\title{
Integer Charge Transfer and Hybridization at an Organic Semiconductor / Conductive Oxide Interface
}

Marco Gruenewald, Laura K. Schirra, Paul Winget, Michael Kozlik, Paul F. Ndione, Ajaya K Sigdel, Joseph J Berry, Roman Forker, Jean-Luc Bredas, Torsten Fritz, and Oliver L.A. Monti

J. Phys. Chem. C, Just Accepted Manuscript • DOI: 10.1021/jp512153b • Publication Date (Web): 11 Feb 2015

Downloaded from http://pubs.acs.org on February 22, 2015

\section{Just Accepted}

"Just Accepted" manuscripts have been peer-reviewed and accepted for publication. They are posted online prior to technical editing, formatting for publication and author proofing. The American Chemical Society provides "Just Accepted" as a free service to the research community to expedite the dissemination of scientific material as soon as possible after acceptance. "Just Accepted" manuscripts appear in full in PDF format accompanied by an HTML abstract. "Just Accepted" manuscripts have been fully peer reviewed, but should not be considered the official version of record. They are accessible to all readers and citable by the Digital Object Identifier (DOI®). "Just Accepted" is an optional service offered to authors. Therefore, the "Just Accepted" Web site may not include all articles that will be published in the journal. After a manuscript is technically edited and formatted, it will be removed from the "Just Accepted" Web site and published as an ASAP article. Note that technical editing may introduce minor changes to the manuscript text and/or graphics which could affect content, and all legal disclaimers and ethical guidelines that apply to the journal pertain. ACS cannot be held responsible for errors or consequences arising from the use of information contained in these "Just Accepted" manuscripts. 


\title{
Integer Charge Transfer and Hybridization at an
}

\section{Organic Semiconductor / Conductive Oxide}

\author{
Interface \\ Marco Gruenewald ${ }^{\dagger, 1}$, Laura K. Schirra ${ }^{\dagger, 2}$, Paul Winget ${ }^{\dagger, 3}$, Michael Kozlik ${ }^{1}$, Paul F. Ndione ${ }^{4}$, \\ Ajaya K. Sigdel ${ }^{4}$,Joseph J. Berry ${ }^{4}$, Roman Forker ${ }^{1}$, Jean Luc Brédas ${ }^{* 3,5}$, Torsten Fritz ${ }^{*}$, , and \\ Oliver L. A. Monti ${ }^{*}, 2$ \\ ${ }^{1}$ Friedrich Schiller Universität Jena, Institut für Festkörperphysik, Helmholtzweg 5, 07743 Jena, \\ Germany \\ ${ }^{2}$ Department of Chemistry and Biochemistry, The University of Arizona, 1306 E. University \\ Blvd., Tucson, AZ, 85721, USA \\ ${ }^{3}$ School of Chemistry and Biochemistry and Center for Organic Photonics and Electronics, \\ Georgia Institute of Technology, Atlanta, Georgia, 30332-0400, USA \\ ${ }^{4}$ National Renewable Energy Laboratory, Golden, Colorado, 80401, USA \\ ${ }^{5}$ Solar and Photovoltaics Engineering Research Center, King Abdullah University of Science \\ and Technology - KAUST, Thuwal 23955-6900, Kingdom of Saudi Arabia \\ e-mail: monti@u.arizona.edu, torsten.fritz@uni-jena.de, jean-luc.bredas@kaust.edu.sa \\ Tel.: ++15206261177 \\ ${ }^{\dagger}$ These authors contributed equally to this work.
}

\section{KEYWORDS}

RECEIVED DATE 


\begin{abstract}
We investigate the prototypical hybrid interface formed between PTCDA and conductive ndoped $\mathrm{ZnO}$ films by means of complementary optical and electronic spectroscopic techniques. We demonstrate that shallow donors in the vicinity of the $\mathrm{ZnO}$ surface cause an integer charge transfer to PTCDA, which is clearly restricted to the first monolayer. By means of DFT calculations, we show that the experimental signatures of the anionic PTCDA species can be understood in terms of strong hybridization with localized states (the shallow donors) in the substrate and charge back-donation, resulting in an effectively integer charge transfer across the interface. Charge transfer is thus not merely a question of locating the Fermi level above the PTCDA electron-transport level, but requires rather an atomistic understanding of the interfacial interactions. The study reveals that defect sites and dopants can have a significant influence on the specifics of interfacial coupling and thus on carrier injection or extraction.
\end{abstract}


Grünewald et al.

J. Phys. Chem. C

\section{INTRODUCTION}

The interface between organic semiconductors and electrodes plays a central role in organicbased optoelectronics and next-generation energy conversion technologies, since they control charge collection and charge injection. Specifically, the organic / metal interface has been the subject of intense study: Exchange correlation effects, chemisorption vs. physisorption, local electric fields and gap states, among others, are considered to be key factors in establishing energy-level alignment and ultimately carrier dynamics. ${ }^{1-6}$ In contrast, interfaces between organic semiconductors and transparent conductive oxides (TCOs) have received less attention. Compatible with the properties of organic semiconductors, TCOs function as low-temperature processable semiconductors and are used as charge-selective interlayers or transparent electrodes. $^{7-9}$ In comparison to metal surfaces, the complex surface chemistry of TCOs, much reduced screening, and the presence of a bandgap, alter interfacial coupling pathways and mechanisms. However, a molecular level understanding of the interfacial electronic structure at such hybrid interfaces is still far from being complete. ${ }^{10}$

Due to its abundance and facile solution processing, $\mathrm{ZnO}$ films have received renewed attention in hybrid organic / inorganic electronics. ${ }^{11-14}$ Several studies have also investigated specific organic / $\mathrm{ZnO}$ interfaces, both theoretically and experimentally. ${ }^{15-18}$ Winget et al. previously reported the formation of interface states at an organic / $\mathrm{ZnO}$ interface, attributed to the presence of specific gap states in highly conductive $\mathrm{ZnO}$ films. ${ }^{19}$ Indeed, electronic coupling between molecules and the $\mathrm{ZnO}$ surface has also been shown to determine excited-state dynamics at these interfaces. ${ }^{20-22}$ The precise nature of these newly formed interface states is generally difficult to establish, making rational control of such interfaces still a significant 
challenge. ${ }^{23}$ Indeed, seemingly different conceptual models are frequently involved in discussing the formation of interfacial states, and the distinction between hybridization and other forms of coupling at the interface are usually not clear. This is not least due to the limited ability of photoelectron spectroscopies to distinguish easily between different interfacial interactions.

Here, we present a detailed study that combines differential reflectance spectroscopy (DRS), ultraviolet and x-ray photoemission spectroscopies, and first-principles electronic-structure calculations of the hybrid interface between the prototypical organic acceptor perylene-3,4,9,10tetracarboxylic dianhydride (PTCDA) and conductive n-type $\mathrm{ZnO}$ thin films. With this combined approach, we are able to identify unambiguously the origin of the observed interface state and discuss the mechanism for its formation in relation to reports of charge transfer states observed at organic / metal interfaces. Our results point to an integer charge transfer mechanism from the $\mathrm{ZnO}$ surface to the first PTCDA monolayer. Further, we find that contributions from backdonation have to be taken into account as well to understand the charge redistribution.

\section{EXPERIMENTAL METHODS}

Materials: Two different types of $\mathrm{ZnO}$ thin films were grown in order to meet the specific requirements of the different analysis techniques. The thin films used for photoelectron spectroscopy have been described in detail elsewhere. ${ }^{19}$ Briefly, atomic layer deposition (ALD) on ITO or pulsed laser deposition on c- and r-plane sapphire was used to prepare n-doped films with an estimated defect concentration of $10^{19}-10^{20} \mathrm{~cm}^{-3}$. On ITO and c-plane sapphire, the resulting polycrystalline films were oriented towards the (0002) surface, while (1120) was preferred on r-plane sapphire. For PTCDA and all other systems investigated, ${ }^{19,23}$ both x-ray and ultraviolet photoelectron spectra were found to be insensitive to preferred crystalline orientation 


\section{Grünewald et al.}

or growth method. For DRS, it was necessary to remove interlayers in order to prevent strong optical interference effects that precluded spectroscopic analysis. Therefore, $30 \mathrm{~nm} \mathrm{ZnO}$ was sputter-deposited on quartz glass at room temperature, with a deposition rate of $5 \mathrm{~nm} / \mathrm{min}$ and using an argon flow at $6 \mathrm{sccm}$. During deposition, the pressure was kept at approx. $3 \cdot 10^{-3}$ mbar. After introduction into the respective ultrahigh vacuum chambers, all $\mathrm{ZnO}$ films were gently sputtered with $\mathrm{Ar}^{+}(1 \mathrm{kV}, 100 \mathrm{nA}, 15 \mathrm{~min})$. As detailed elsewhere, ${ }^{23}$ this treatment primarily removes adventitious adsorbates with mild enrichment for $\mathrm{Zn}$ in the near surface region, and no indication of $\mathrm{Zn}$ segregation or surface metallization could be observed in the ultraviolet photoelectron spectra. $^{24}$ PTCDA was evaporated from a Knudsen cell at 0.2 monolayer equivalents (MLE) / min, with $3.2 \AA$ corresponding to 1 MLE.

DRS: The experimental set-up for DRS has been described already elsewhere. ${ }^{25-27}$ Briefly, the photon energy- and film thickness-dependent reflectance was measured in situ in ultrahigh vacuum. The optical functions of the glass substrate and the bare $\mathrm{ZnO}$ film were determined separately by transmission and reflectance measurements in an ex situ Varian Cary 5000 spectrophotometer. Subsequently, the contribution of PTCDA to the DR spectra and the complex dielectric function was extracted using a numerical algorithm introduced detailed previously. ${ }^{25,28}$

Photoelectron spectroscopy: As already described elsewhere, valence band ultraviolet photoemission spectra (UPS) were obtained with a He(I) lamp in ultrahigh vacuum (base pressure $2 \cdot 10^{-10} \mathrm{mbar}$, with photoelectrons collected normal to the surface with an acceptance angle of $\pm 12.5^{\circ}$ and an applied sample bias of $-5 \mathrm{~V}$. The workfunction is obtained from the secondary electron cut-off in UPS. Binding energies for the molecular features are reported as peak onset, determined by the intercept of the slope at the inflection point of the peak and the baseline. This reflects most accurately the relevant transport level by diminishing dispersion 
Grünewald et al.

J. Phys. Chem. C

effects, incomplete screening of the photohole and peak asymmetries introduced by inelastic scattering. ${ }^{29}$ All results presented here are insensitive to the specific $\mathrm{ZnO}$ film growth protocol.

Computational: Electronic structure calculations were performed using the Vienna ab initio simulation package (VASP) ${ }^{30}$ at the generalized gradient approximation level, using PBE and including a Hubbard $U$ correction $(U=8.5 \mathrm{eV})$. This approach has been detailed and validated extensively already, and as discussed previously yields qualitatively similar results as the computationally more involved approaches using range-separated functionals despite non-trivial issues of error-cancellation. ${ }^{10,19,23,31}$ Both polar and non-polar surfaces were investigated computationally; in agreement with experiment, the conclusions do not differ substantially in response to the specific surface orientation, ${ }^{19,23}$ allowing us to concentrate here mainly on the prototypical (10̄10) surface to elaborate the salient features of the PTCDA / ZnO interface. We computed the electronic structure for an orthogonal unit cell of ten $\mathrm{ZnO}$ layers with dimensions of $19.50 \AA \times 10.41 \AA$ in the [1120] and [0001] directions and $36.00 \AA$ in the [10 $\overline{1} 0]$ direction, with PTCDA molecules adsorbed in rows at a resulting monolayer density of $4.92 \times 10^{13}$ molecules $\mathrm{cm}^{-2}$. Stoichiometric surfaces lead to qualitatively wrong workfunctions and energylevel alignment, as already reported for the bare surfaces and interfaces with perylene bisimides. ${ }^{19,31}$ Instead, shallow donor levels such as $\mathrm{Zn}_{\mathrm{i}}$ are necessary to obtain good qualitative and reasonable quantitative agreement between experiment and theory. $\mathrm{Zn}_{\mathrm{i}}$ shift the chemical potential and may couple directly to the molecular adsorbate if in close proximity to the surface. ${ }^{19}$ For this reason, a concentration of $1 \mathrm{Zn}_{\mathrm{i}}$ out of $120 \mathrm{Zn}$ atoms was introduced near the surface in the calculations, leading to a free electron density in the near-surface region of $1 \cdot 10^{14}$ electrons $\mathrm{cm}^{-2}$, in agreement with the level of n-doping found in the thin films and relevant for potential direct coupling pathways with the adsorbate. 
Grünewald et al.

J. Phys. Chem. C

\section{RESULTS}

In order to investigate the molecular interactions at the interface with n-doped conductive $\mathrm{ZnO}$ films, we employed DRS that provides access to the wavelength-dependent and coveragedependent complex dielectric function of PTCDA. DRS is defined as:

$$
D R S(E, d) \equiv \frac{\Delta R}{R}:=\frac{R(E, d)-R(E, d=0)}{R(E, d=0)}
$$

where $R(E, d)$ denotes in our case the reflectivity of the sample stack consisting of the quartz glass substrate, $30 \mathrm{~nm} \mathrm{ZnO}$, and a thin PTCDA layer of varying thickness. Consequently, the DRS signal depends on the photon energy $E$ and the PTCDA film thickness $d$. The raw DR spectra are shown in the Supplementary Figure 1, for coverages ranging from 0 to 7 MLE of PTCDA on $\mathrm{ZnO}$ on glass, with increments of 0.2 MLE in the range of $0-1$ MLE. In Figure 1(a) we present the imaginary component of the dielectric function $\varepsilon^{\prime \prime}$ of the adsorbate film extracted from the DRS data, which represents the absorption behavior, following the numerical procedure outlined previously. ${ }^{25}$ 
a)

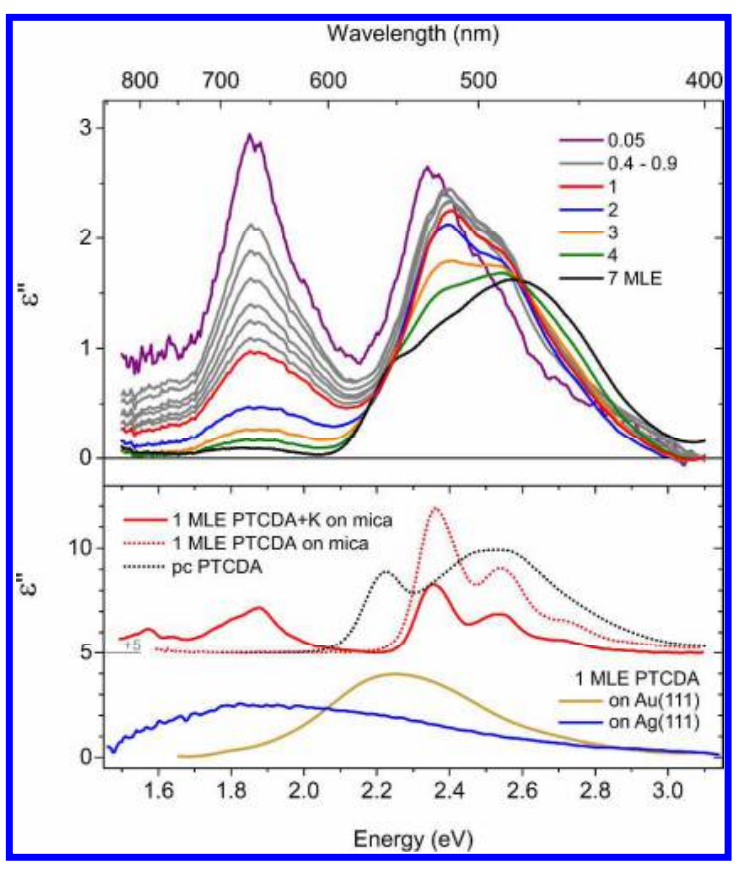

b)

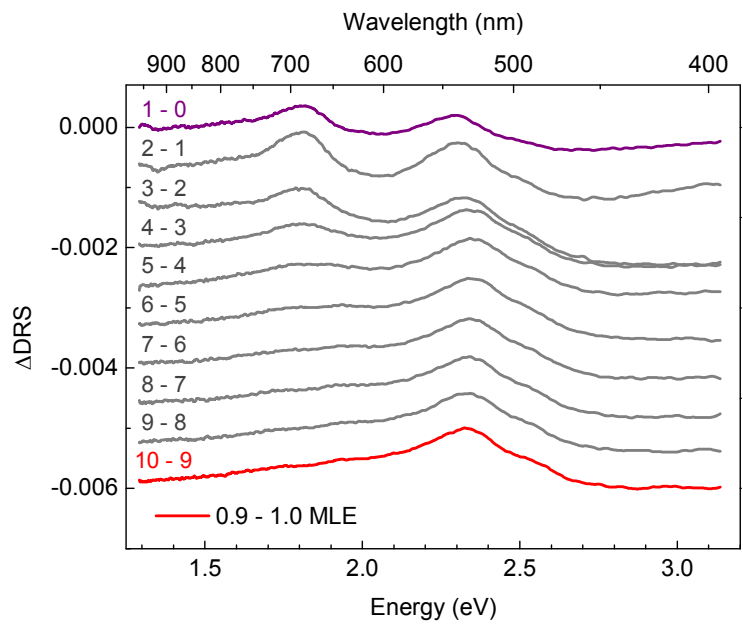

Figure 1 a) Imaginary part $\varepsilon^{\prime \prime}$ of the dielectric function of PTCDA on $30 \mathrm{~nm} \mathrm{ZnO}$ on quartz glass for nominal thicknesses ranging from submonolayers up to 7 MLE extracted from the DRS shown in Supplementary Figure 1. For comparison $\varepsilon^{\prime \prime}$ spectra of 1 MLE PTCDA on inert mica, ${ }^{27}$ 1 MLE of PTCDA partially potassium-doped providing pronounced new features of PTCDA radical anions at low photon energies (investigated in detail previously ${ }^{32}$ ), polycrystalline (pc) PTCDA $^{33}$ and the dramatically broadened spectra of 1 MLE of PTCDA on Au(111) and $\operatorname{Ag}(111)^{34,35}$ are shown in the bottom panel. b) $\triangle D R S$ of PTCDA on quartz glass for nominal thicknesses ranging from submonolayers up to 1 MLE. The graphs are labeled according to the scheme $(m+1)-m$ referring to eq. (2).

At $\hbar \omega \geq 2.1 \mathrm{eV}$, the spectra display the typical PTCDA $\mathrm{S}_{0} \rightarrow \mathrm{S}_{1}$ absorption for all coverages. For the thinnest films the vibronic progression typical of monomeric PTCDA molecules is visible, and is consistent with what is observed in solution and thin films on mica, although somewhat affected by inhomogeneous broadening and/or weak hybridization with the surface (cf. discussion section); this observation indicates that there are no appreciable interactions among the molecular transition dipoles. ${ }^{32,36}$ At higher coverages, the formation of PTCDA molecular stacks (or at least piles) is expected. The interactions among molecular 
transition dipoles in such stacks changes the spectral shape noticeably, and the bulk spectrum of PTCDA emerges. ${ }^{36}$ It is worthwhile to note that the convergence towards the bulk spectrum takes as much as seven MLEs due to the growth of three-dimensional clusters of disordered PTCDA on $\mathrm{ZnO}$, in contrast to the case on mica where four MLEs are sufficient.

The most striking feature in the DR spectra appears, however, at an energy of $1.86 \mathrm{eV}$, well below the absorption bands of neutral PTCDA monomers and the spectral features observed in bulk PTCDA. ${ }^{37}$ A comparison with DR spectra of thin films of PTCDA on mica, as deposited and potassium-doped, is shown in the bottom panel of Figure 1(a), and identifies this feature unequivocally as originating from PTCDA radical anions $\left(\mathrm{PTCDA}^{\bullet-}\right)$. Exposure of robust molecules such as PTCDA to a potassium vapor induces integer ground-state charge transfer to form $\mathrm{K}^{+}$and $\mathrm{PTCDA}^{\bullet-}$, where the latter exhibits an absorption band at $1.86 \mathrm{eV}$ with a peak shape similar to that observed on $\mathrm{ZnO}$. We conclude therefore that the growth of PTCDA on $\mathrm{ZnO}$ leads directly to the formation of $\mathrm{PTCDA}^{\bullet}$, carrying a full additional electron, without any need for doping. This is in contrast to PTCDA adsorbed on mica where intentional doping with $\mathrm{K}$ is required to produce the PTCDA anion. ${ }^{32}$

In order to investigate the origin of the PTCDA radical anion in more detail, we plot the $\triangle D R S$ spectra obtained by subtracting pairs of reflectance spectra $R_{m}$ and $R_{m+1}$ representing two sequential coverages:

$$
\Delta D R S_{(m+1)-m}:=\frac{R_{m+1}-R_{m}}{R_{m}}
$$

In contrast to $D R S$ itself, where all absorbing species in the layer at a specific total thickness $d$ contribute to the signal, $\triangle D R S$ allows one to clearly determine which absorbing species are emerging or vanishing in the small thickness increment from $d_{\mathrm{m}}$ to $d_{\mathrm{m}+1}$. 


\section{Grünewald et al.}

The $\Delta D R$ spectra obtained for the data in Figure 1(a) are shown in Figure 1(b). Strikingly, the anion band is most prominent at coverages of $\leq 1$ MLE, weakening already below 1 MLE, and is essentially absent above that coverage. Indeed, close inspection of these spectra reveals that anion formation is restricted to coverages of $\leq 0.5$ MLE, after which additional PTCDA molecules grow as neutral molecules on the surface. These spectra demonstrate that the strong acceptor PTCDA undergoes ground state charge transfer at the interface with conductive n-ZnO thin films: already below $1 \mathrm{MLE}$, the $\mathrm{PTCDA}^{\bullet-}$ absorption has saturated and no further increase is observed, identifying the PTCDA anion as a strictly interfacial species.

We note that the observed ground-state charge transfer is not to be confused with conventional band doping: In PTCDA layers with a coverage below 1 MLE, no bands are formed. Consequently, one has to understand the charge transfer as charging of single quantum objects, i.e., single molecules, by an additional full electron rather than as doping of a (3dimensional) solid where a fractional charge per building block is the usual case.

The DR spectra can be separated into two regions: below (above) a threshold energy of $c a$. $2.1 \mathrm{eV}$, the absorption features stem from the PTCDA radical anions (neutral PTCDA molecules). This situation is similar to the K-doping of PTCDA on mica, ${ }^{32}$ where the K-doping of a monolayer of neutral PTCDA initially follows the reaction $\mathrm{K}+\mathrm{PTCDA} \rightarrow \mathrm{K}^{+}+\mathrm{PTCDA}^{\bullet-}$. Hence, for every radical anion formed, a neutral molecule disappears. Consequently, the absorbance below (above) $2.1 \mathrm{eV}$ increases (decreases). Given that the same number of charged or uncharged molecules are responsible for the changes in both spectral regions, the ratio of the oscillator strengths between PTCDA radical anions and neutral molecules can be estimated as the ratio of the change in spectral area below and above this threshold energy for consecutive spectra during K-doping. Similar arguments apply for the absorbance spectra in solution, as 
discussed in previously. ${ }^{32}$ There, discharging of dissolved PTCDA anions causes the low-energy spectral features (property of the PTCDA anions) to decrease while the high-energy absorbance (property of the neutral PTCDA) simultaneously increased. We decompose therefore the spectra in terms of their relative molecular oscillator strengths:

$$
O S=\int_{\text {absorption band }} \varepsilon^{\prime \prime}(E) \cdot E d E
$$

The ratio of oscillator strengths of these two species calculated for the absorption bands in the energy interval considered here has been previously determined to be $\frac{o S_{\text {anions }}}{\text { os }} \approx 0.6 .^{32}$ Consequently, we estimate the fraction of charged molecules with respect to the total number of molecules within the first MLE (cf. Fig 1(a), thick red line) to be 30-40\%.

Further evidence for our interpretation of charge transfer at the interface can be obtained from investigating the electronic structure. Coverage-dependent valence band photoemission spectra for PTCDA on $\mathrm{ZnO}$ are shown in Figure 2. While the spectra generally show the emergence of PTCDA features (e.g., the first ionization feature, "HOMO" at -2.1(1) eV (peak onset), see $\mathrm{SI}$ ) and the suppression of the $\mathrm{O} 2 \mathrm{p}$ bands associated with $\mathrm{ZnO}$, the most striking changes are $(i)$ the rapid increase of the workfunction (Figure 2(a)) and (ii) the emergence of a new spectroscopic feature at $-0.3(1) \mathrm{eV}$ (peak onset), located in the bandgaps of both PTCDA and $\mathrm{ZnO}$ (Figure 2(b)). 

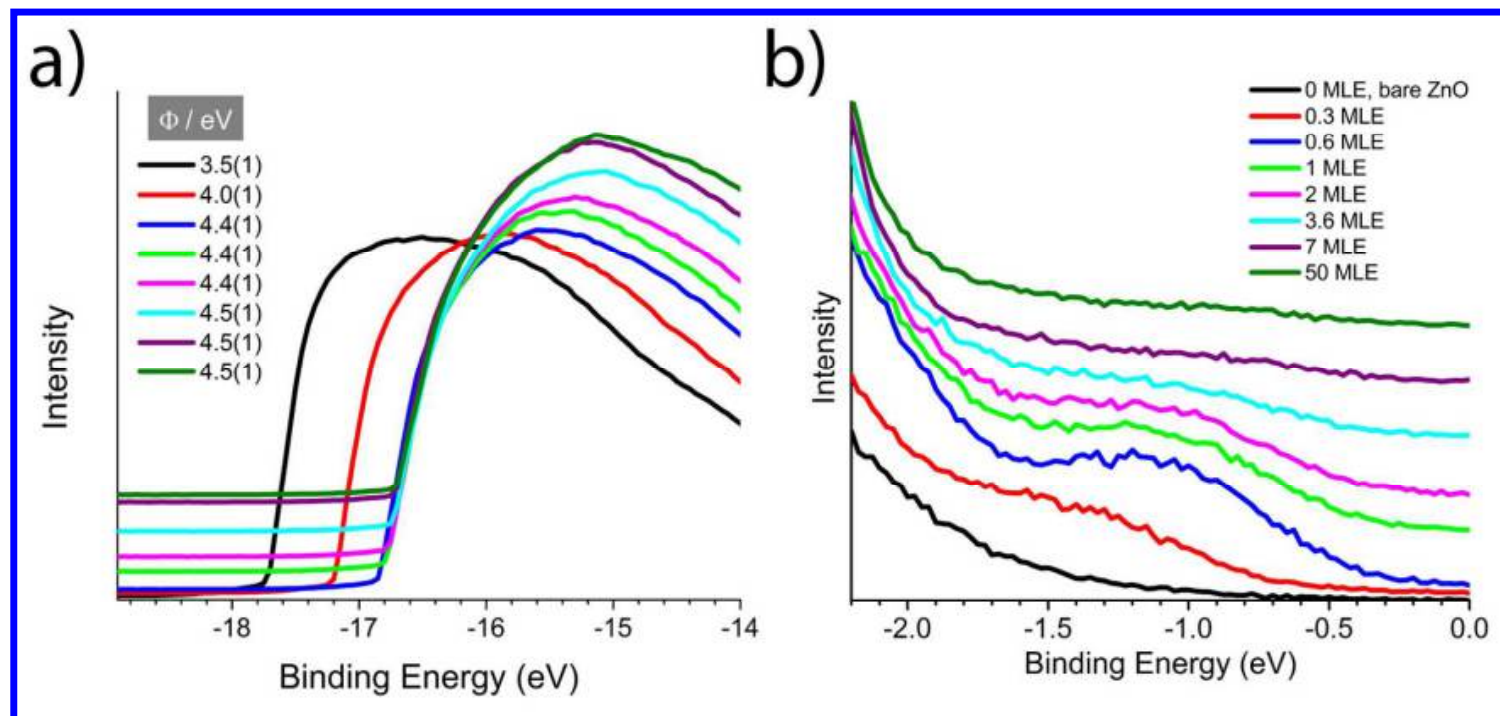

Figure 2 a) Secondary electron cut-off and work function of bare atomic layer deposited $\mathrm{ZnO}$ and thin layers of PTCDA subsequently deposited onto the $\mathrm{ZnO}$ surface. b) Close-up of the interface state peak as a function of coverage. Full spectra are shown in the Supplementary Information.

The workfunction increase indicates a build-up of electron density at the interface, in agreement with ground-state interfacial charge transfer from $\mathrm{ZnO}$ to PTCDA. Remarkably, the workfunction saturates at 0.6 MLE PTCDA already, which is consistent with the observed anion formation at coverages $\leq 0.5 \mathrm{MLE}$ in DRS. The new feature at $-0.3(1) \mathrm{eV}$ also increases up to a nominal coverage of $0.6 \mathrm{MLE}$, before weakening at higher coverages and finally completely disappearing in films with thicknesses in considerable excess of 1 MLE. Due to the finite escape depth of photoelectrons with kinetic energies around $16 \mathrm{eV},{ }^{38}$ a species formed only at the PTCDA / ZnO interface is expected to weaken in UPS at coverages significantly above 1 MLE, identifying this peak as originating from an interfacial process. The binding energy for this state is close to the Fermi energy, $E_{F}$, and located in the bandgap of both $\mathrm{ZnO}$ and neutral PTCDA, indicating charge transfer and formation of $\operatorname{PTCDA}^{\bullet-}$. This is consistent with $(i)$ the observed workfunction increase, (ii) the anion spectrum in DRS, and (iii) a new peak arising in the C $1 \mathrm{~s} \mathrm{x}-$ 
ray photoelectron emission spectrum (XPS, see Supplementary Information). The interfacial electronic structure obtained from UPS and the chemical identification of thin film molecular species in DRS show together in an unambiguous fashion that many of the PTCDA molecules in the first monolayer couple electronically strongly to the conductive $\mathrm{n}-\mathrm{ZnO}$ substrate, resulting in an integer charge transfer to form the PTCDA radical anion.

Remarkably, our observations are independent of the specific $\mathrm{ZnO}$ film growth protocols for these highly n-doped $\mathrm{ZnO}$ films and any variations of the associated preferential crystallographic orientation. This indicates that the interfacial interactions are not caused by a surface - molecule interaction specific to a particular surface termination, but rather by a more general mechanism. This suggests further that our observations are likely more general and extend beyond the particular molecular system and the specific oxide investigated here.

We now turn to electronic-structure calculations in order to uncover a more detailed molecular-level mechanism of this charge transfer. Using a recently established and validated methodology for highly conductive n-type $\mathrm{ZnO}$ films, ${ }^{19,23,31}$ we modeled the bare $\mathrm{ZnO}$ surface and the interface with PTCDA for three different limiting cases: (i) A stoichiometric and thus idealized $\mathrm{ZnO}$ surface, (ii) $\mathrm{ZnO}$ including deep donor oxygen vacancies $\left(\mathrm{O}_{\mathrm{v}}\right)$ often claimed to be responsible for the $\mathrm{n}$-character of $\mathrm{ZnO},{ }^{39,40}$ and (iii) $\mathrm{ZnO}$ containing shallow donors modeled here by zinc interstitials $\left(\mathrm{Zn}_{\mathrm{i}}\right)$ contributing each two electrons, resulting in a free-electron density of $10^{14}$ electrons $\mathrm{cm}^{-2}$. Table 1 shows the workfunction change for these different surfaces upon adsorption of a PTCDA monolayer.

Table 1 Individual contributions $\Delta \mathrm{V}_{\mathrm{SAM}}, \Delta \mathrm{V}_{\text {id }}$ and $\Delta \mathrm{V}_{\mathrm{gr}}$ to computed workfunction changes $\Delta \Phi$ and amount of charge transfer $\mathrm{q}$ at the interface of 1 monolayer PTCDA with stoichiometric $\mathrm{ZnO}, \mathrm{ZnO}$ with oxygen vacancies $\mathrm{O}_{\mathrm{v}}$ and $\mathrm{ZnO}$ with zinc interstitials $\left(\mathrm{Zn}_{\mathrm{i}}\right)$. The values shown here pertain to $\mathrm{ZnO}(\mathbf{1 0} \overline{\mathbf{1}} \mathbf{0})$. For an explanation of $\Delta \Phi$, $\Delta \mathrm{V}_{\mathrm{SAM}}, \Delta \mathrm{V}_{\mathrm{id}}, \Delta \mathrm{V}_{\mathrm{gr}}$ and $\mathrm{q}$ see text. 


\begin{tabular}{|c|c|c|c|c|c|}
\hline & $\Delta \Phi(\mathbf{e V})$ & $\Delta \mathbf{V}_{\text {SAM }}(\mathbf{e V})$ & $\Delta \mathbf{V}_{\text {id }}(\mathbf{e V})$ & $\Delta \mathbf{V}_{\mathbf{g r}}(\mathbf{e V})$ & $\mathbf{q}(\mathbf{e})$ \\
\hline $\begin{array}{c}\text { PTCDA on } \mathrm{ZnO} \\
\text { (stoich.) }\end{array}$ & -0.76 & -0.30 & -0.37 & -0.06 & 0.02 \\
\hline $\begin{array}{c}\text { PTCDA on } \mathrm{ZnO} \\
\left(\mathrm{O}_{\mathrm{V}}\right)\end{array}$ & -0.24 & -0.33 & 0.31 & -0.25 & -0.35 \\
\hline $\begin{array}{c}\text { PTCDA on } \mathrm{ZnO} \\
\left(\mathrm{Zn}_{\mathrm{i}}\right)\end{array}$ & +1.18 & -0.39 & 1.59 & -0.24 & -0.98 \\
\hline
\end{tabular}

For these ideal monolayer interfaces with $\mathrm{ZnO}$, workfunction changes for the stoichiometric and $\mathrm{O}_{\mathrm{v}}$ surfaces are both qualitatively and quantitatively different from the rapid workfunction increase of 1.0(1) eV observed experimentally. In contrast, the inclusion of shallow donors such as $\mathrm{Zn}_{\mathrm{i}}$ results in good agreement between theory and experiment which is consistent with the findings for perylene bisimide on $\mathrm{ZnO} .{ }^{19}$ We note that this agreement also extends to the bare $\mathrm{ZnO}$ surfaces, where $\mathrm{Zn}_{\mathrm{i}}$ species are necessary for agreement with the experimentally observed low workfunction, regardless of the specific surface orientation modeled. ${ }^{19} \mathrm{ZnO}$ thin films that are useful as carrier-selective interlayers in hybrid organic/inorganic electronics include a considerable concentration of shallow donors in order to exhibit the desirable high conductivity levels. Indeed, some of the films used in the experiments are nearly degenerately doped with a carrier concentration of $10^{19}-10^{20} \mathrm{~cm}^{-3}$.

The workfunction change arises from several separate contributions that provide further insight into the interfacial interactions: ${ }^{41}$

$$
\Delta \Phi=\Delta V_{S A M}+\Delta V_{i d}+\Delta V_{g r}
$$

where $\Delta V_{S A M}$ is the electrostatic potential drop across the molecular layer suspended in vacuum while maintaining the geometry of the thin film on the surface, $\Delta V_{i d}$ is the charge rearrangement- 
induced interface dipole arising from adsorption of PTCDA on $\mathrm{ZnO}$, and $\Delta V_{g r}$ captures the potential change due to geometric rearrangement upon molecular adsorption. Table 1 lists these values for all three variants of the $(10 \overline{1} 0)$ surface. Clearly, structural relaxations upon adsorption and the electrostatic potential arising from the PTCDA layer contribute minimally to the calculated workfunction change, particularly for the defect containing surface. This effect is dwarfed by the interface dipole contribution $\Delta V_{i d}$ which dominates the workfunction change and is responsible for the observed workfunction increase upon adsorption of a PTCDA monolayer. This interface dipole originates from charge reorganization at this interface and results in a net charge transfer $q$ across the interface, tabulated in the last column of Table 1. Strikingly, for the $\mathrm{ZnO}$ surface with shallow donors $\left(\mathrm{Zn}_{\mathrm{i}}\right)$, almost a full electron is transferred from $\mathrm{ZnO}$ to PTCDA at monolayer coverage. We note that these conclusions remain unaltered even in the event of adsorption to a hydrated surface, albeit to a somewhat smaller extent (-0.44 e). This finding supports the observation of an anionic interface state of PTCDA on $\mathrm{ZnO}$ as observed with both DRS and UPS.

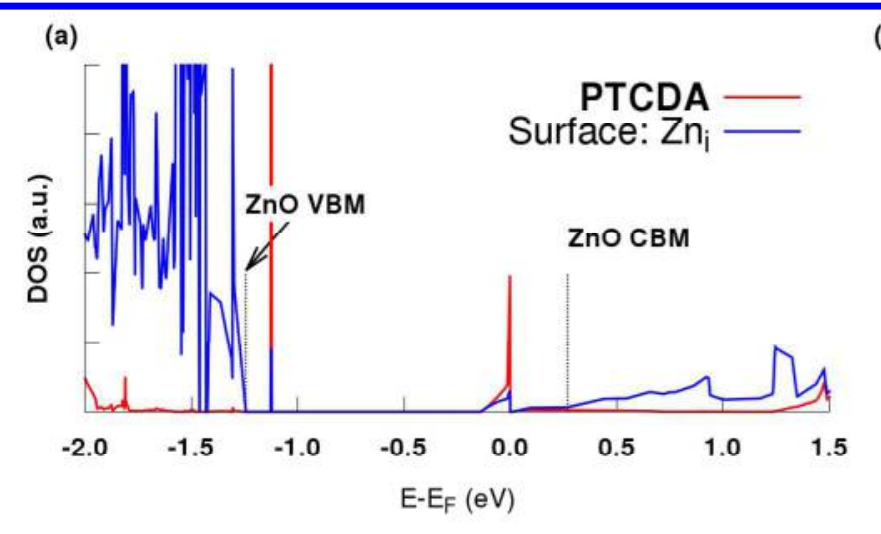

(b)

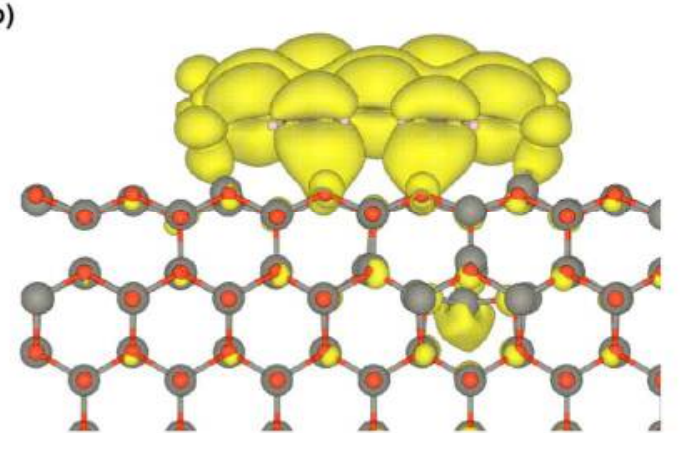

Figure 3 a) Projected density of states for $\mathrm{ZnO}$ (1010) containing $\mathrm{Zn}_{\mathrm{i}}$ (blue dotted line) and for PTCDA (red solid line). b) Electron density plot of interface state located near $E_{F}$, comprised of a LUMO-like wavefunction and weakly hybridized with the $\mathrm{Zn}_{\mathrm{i}}$. $\mathrm{C}$ atoms are black, $\mathrm{H}$ atoms grey, $\mathrm{O}$ atoms red and $\mathrm{Zn}$ atoms silver. 
The effect of electron transfer to PTCDA is also clearly visible in the projected density of states (pDOS), shown for the $\mathrm{Zn}_{\mathrm{i}}$ surface in Figure 3(a). We recall that despite the underestimation of the bandgaps of both $\mathrm{ZnO}$ and PTCDA, our computational approach represents the relative energy-level alignment at this interface well as detailed elsewhere. ${ }^{19,23}$ With $\mathrm{Zn}_{\mathrm{i}}$ present, the HOMO is located inside the $\mathrm{ZnO}$ bandgap as observed experimentally, and a new state appears close to $E_{F}$. This state corresponds to the interface state observed in UPS. A plot of the calculated electron density of this state readily reveals its identity (Figure 3(b)) as containing strong PTCDA LUMO (lowest unoccupied molecular orbital) character, as expected for charge transfer from $\mathrm{ZnO}$ to PTCDA. Interestingly, there is as well a contribution from $\mathrm{Zn}_{\mathrm{i}}$ highlighting the hybridized character of this state.

\section{DISCUSSION}

Before analyzing our findings further and in order to connect our work to the gamut of other interacting adsorbate systems, it is important to differentiate clearly between two different limiting cases of surface / molecule interactions. In particular, we highlight the difference between $i$ ) strong hybridization with delocalized substrate states and ii) weak molecule substrate interaction, i.e., no hybridization, presented here as two well-known and widely appreciated extreme cases of coupling to the substrate. The basic schemes are sketched in Figure 4. In cases where there is no hybridization, the molecule remains almost unchanged with regard to its orbital structure (Fig. 4(a)). Due to the essentially two-dimensional nature of the interface and poor intermolecular coupling, no new bands electronic develop. As a consequence, the molecules retain their character as individual nano objects, and only integer charge transfer is feasible in 
this limit. This leads to distinct charge-states and different orbital filling schemes of the charged single nano objects, and in turn to strongly altered optical absorption spectra.

In contrast, the charge transfer mechanism upon strong hybridization with delocalized substrate states like on metals is entirely different (Fig. 4(b)): Here, the strong hybridization leads to a substantial broadening of molecular states. ${ }^{42,43}$ Further, the intermolecular interaction is mediated by the substrate such that new electronic bands can be formed even in a twodimensional film with otherwise weak intermolecular coupling. ${ }^{44}$ Accordingly, the molecules lose their individual character. From a simplified point of view, this can be regarded as formation of a new system at the interface consisting of the original molecules and a certain number of strongly coupled substrate atoms. This situation leads to rather broad and unstructured optical absorption bands usually combined with a strong spectral shift. ${ }^{34,35,45}$ Consequently, the resulting spectra bear no resemblance to the monomer spectra (cf. Fig. 1(a), lower panel). Note that the apparent spectral width depends on the strength of molecule- metal interaction and is therefore a fingerprint of the degree of chemisorption vs. physisorption. ${ }^{35}$

Yet another general consequence of strong hybridization is the effect of charge backdonation from the molecule toward the substrate, frequently observed in strong hybridization at metal / organic interfaces: Due to broadening of the molecular states, certain deep lying orbitals extend energetically above the Fermi level, such that the charge density on the molecule is lowered, as was explicitly shown for the case of PTCDA on $\operatorname{Ag}(111) .{ }^{46}$ All these effects (spatial extension of the molecular wave function into the substrate, charge back-donation, formation of two-dimensional bands at the interface due to the substrate-mediated intermolecular interaction) result in a fractional net charge transfer, usually well below 1 electron charge and e.g., in the case of PTCDA on $\mathrm{Ag}(111)$ as low as 0.3 e. $^{46}$ 
These two limiting cases permit us to shed more light into the specific interactions at the PTCDA / ZnO interface. Investigating small contributions of hybridization by our optical spectroscopic method presents a challenge, since hybridized interface states may not be easily interpreted by comparison with simple chemical species in bulk or solution. As a general trend, however, we observed in previous DRS experiments for molecules on metal substrates a broadening of the spectra depending on the strength of electronic interaction (see lower panel of Fig. 1(a)), characteristic of strong hybridization with delocalized states as delineated above. ${ }^{35}$ Therefore, the slight broadening of the anion peaks in the DRS spectra in Figure 1(a) at higher deposition levels may be partially attributed to only a weak hybridization of the PTCDA 
molecules on $\mathrm{ZnO}$. On the scale of electronic interaction strength, the adsorption regime can be however clearly regarded as physisorption, comparable to PTCDA on top of 1 ML PTCDA / $\operatorname{Ag}(111)$, where a slight broadening of the optical spectra is also observed. ${ }^{35}$ In that case, the $1^{\text {st }}$ ML PTCDA, in direct contact with the silver substrate, is strongly hybridized and functions as decoupling interlayer for the subsequent PTCDA layer, which is consequently only slightly hybridized. This suggests initially that PTCDA on ZnO falls into the limit of weak hybridization and expected integer charge transfer, as borne out by an initial analysis of the computational results $(0.98$ e transferred to PTCDA).

There is however a notable discrepancy between the computational results and the DRS data regarding the extent of charge transfer: By analyzing the oscillator strengths of both anion and neutral PTCDA transitions in DRS, we obtain an average charge transfer of approximately 1 electron per 3 molecules for 1 MLE, and even more for submonolayers of PTCDA, where we find an average of up to 1 electron per 2 molecules at 0.5 MLE and where the anion signature in UPS also reaches its maximum. In contrast, DFT predicts transfer of almost a full electron per molecule at one monolayer coverage for the PTCDA / $\mathrm{Zn}_{\mathrm{i}} / \mathrm{ZnO}$ system. We stress, however, that the DRS clearly indicates integer charge transfer to the molecule, i.e., the charged molecules exhibit spectra that appear like anions, consistent with carrying a full charge of one electron per charged molecule and as expected for the weak hybridization limit.

The quantitative discrepancy between experiment and theory can be understood by considering that, contrary to the ideal epitaxial PTCDA growth on $\mathrm{ZnO}$ considered in the DFT calculations, the film structure is likely disordered on the polycrystalline $\mathrm{ZnO}$ films used in our experiments (see atomic force microscopy image, Figure 4 in SI), an assumption consistent with the broadened DR spectra for the $\mathrm{S}_{0} \rightarrow \mathrm{S}_{1}$ absorption of neutral PTCDA. This has two 
consequences: First, the measured coverages do not reflect the actual number of molecules in direct contact with the $\mathrm{ZnO}$ surface, nor are all surface sites that can participate in charge transfer necessarily accessed by PTCDA. As a result, the observed average extent of charge transfer across this interface is lower than estimated for the calculated idealized system, and $\sim 0.33$ electrons per molecule may simply indicate that every $3^{\text {rd }}$ molecule has experienced a full oneelectron reduction by $\mathrm{ZnO}$, as predicted by DFT. Second, the DFT calculations show that the charge transfer is not site specific but orientation-dependent, with the amount of charge transferred reduced when the molecules are rotated (e.g. by $45^{\circ}$ ) in-plane on the surface. A mixture of orientations would be expected on a disordered surface. In both scenarios the observed charge transfer is integer, giving rise to a fully negatively charged molecule in accordance with our interpretation of the DR spectral signature, while the inhomogeneous experimental PTCDA film structure and polycrystalline nature of the $\mathrm{ZnO}$ film does not match the idealized case considered in the computational model. The observed UP and DR spectra can consequently be understood to represent the electronic structure of a supramolecular entity consisting of an ionized shallow donor site such as $\mathrm{Zn}_{\mathrm{i}}$ and a reduced PTCDA molecule.

Charge transfer from an oxide surface to a strong acceptor such as PTCDA is however not merely a question of locating the Fermi level above PTCDA electron-transport levels. In fact, it also involves a measure of rather strong interface hybridization leading to charge back-donation. This can be seen by closer inspection of the UPS and DFT results: By integrating the charge density for the LUMO-like state near the Fermi level (Fig. 3) and assigning the charge based on the Bader volumes for that state, we find a charge of $\sim 2$ e residing in this LUMO-like state of PTCDA. Of these $\sim 2$ e, $\sim 0.6$ e are in fact located well within the $\mathrm{ZnO}$ slab, leaving $\sim 1.4$ e on PTCDA (see Fig. 3(b)). This is still larger than the total charge of 0.98 e transferred to PTCDA 
(see Table 1). This apparent contradiction can be readily understood by taking charge backdonation into account: While the PTCDA LUMO is filled due to the high ZnO Fermi level in the presence of shallow donors, charge $(\sim 0.4$ e) flows back from PTCDA to $\mathrm{ZnO}$ out of a set of lower lying occupied molecular orbitals. Such back-donation is characteristic of strong hybridization and reminiscent of the case of PTCDA adsorbed on the (111) surfaces of coinage metals, ${ }^{46-48}$ as well as, e.g., $\mathrm{F}_{4}-\mathrm{TCNQ}$ on $\mathrm{Cu}(111)$ and $\mathrm{Ag}(111) .{ }^{49,50}$

This interpretation is also fully consistent with the photoelectron spectra: The interface state is not located at exactly $\mathrm{E}_{\mathrm{F}}$, as would be expected for a simple integer charge transfer / weak hybridization scenario. It is rather bound by $0.3(1) \mathrm{eV}$, in agreement with a more complex interfacial charge-rearrangement and a component of strong hybridization. This interpretation highlights the failure of the one-electron interpretation of photoemission data to capture electron correlation and many-body effects at the interface.

Naturally the question arises why our DR spectra do not indicate strong but rather weak hybridization, seemingly contradicting our conclusions above. However, it is necessary to distinguish not merely the strength of the hybridization but also the nature of the states involved. In case of metal substrates, hybridization occurs with strongly delocalized states which gives rise to the observation of broad and structureless absorption spectra. This results in part from coupling to broad bands with substantially delocalized electrons such as e.g. the sp-bands in coinage metal surfaces. In contrast, hybridization in the case of PTCDA on $\mathrm{ZnO}$ involves strongly localized states in rather narrow bands, such as e.g. the shallow donors (cf. Fig. 3(b)). While the interaction strength in terms of charge transfer and back-dotation is strong, this type of hybridization does therefore not lead to a significant spectral broadening. 
The proposed charge transfer mechanism at the hybrid organic/inorganic interface is thus rather more subtle than what might be expected from merely comparing the molecular electron affinity and the Fermi level. Indeed, even any one spectroscopic approach delivers an incomplete understanding of such interfaces. Instead, we show in our combined experimental and computational study that a hybrid organic/inorganic interface such as PTCDA/ZnO does not fall neatly into either of the two limiting cases: While the net result is one of nearly integer charge transfer and apparent weak hybridization, this appearance is in fact caused in part by a strong hybridization and a back-donation. This is rather different from previous reports of ground state charge transfer, e.g., at metal surfaces, and highlights the need for detailed investigations of the electronic structure of hybrid organic/inorganic interfaces. We suggest that this has important consequences for charge transfer dynamics across this interface.

\section{CONCLUSION}

Taken together, the combination of in situ optical spectroscopy with measurements of the interfacial electronic structure and computational modeling of the defective $\mathrm{ZnO}$ surface yields a comprehensive understanding of the prototypical hybrid interface formed between PTCDA and conductive n-doped $\mathrm{ZnO}$ films. Our data show unambiguously that shallow donors cause integer charge transfer to PTCDA, manifesting itself as a new feature in DRS with an absorption band similar to that of PTCDA radical anions, and as a strong workfunction increase in the first monolayer together with the emergence of new features in UPS and XPS. The DFT results indicate that charge transfer arises from the formation of a hybrid interface state between PTCDA and shallow donor levels, here modeled as $\mathrm{Zn}_{\mathrm{i}}$. Charge transfer from a defective oxide surface to a strong acceptor such as PTCDA is therefore not merely a question of locating the Fermi level above the PTCDA electron-transport levels but also involves a measure of interface 
Grünewald et al.

J. Phys. Chem. C

hybridization. Furthermore, back-donation of electrons through occupied molecular orbitals lying below the Fermi energy needs to be taken into account for understanding the charge redistribution at the interface. Our data highlight the important differences between the case of strong hybridization with delocalized states, involving the loss of molecular identity and typically fractional charge transfer, and other forms of interfacial coupling that may accommodate transfer of a full elementary charge and yet also involve strong hybridization, albeit with localized states.

This study opens new avenues to understanding the interfacial electronic structure in the limit of weak screening and suggests that defect sites and dopants can have a significant influence on the specifics of interfacial coupling and thus carrier injection at hybrid interfaces.

\section{ACKNOWLEDGMENT}

M.G., M.K., R.F., and T.F. acknowledge financial support by the Deutsche Forschungsgemeinschaft through grant no. FR875/9-3. This work was also supported as part of the Center for Interface Science: Solar Electric Materials (CISSEM), an Energy Frontier Research Center funded by the U.S. Department of Energy, Office of Science, Basic Energy Sciences under Award Number DE-SC0001084 (P.W., L.K.S., P.F.N., A.K.S., D.S.G., J.J.B., J.S., H.K., B.K., J.L.B. and O.L.A.M.). The computations reported here were performed at the Georgia Tech Center for Computational Molecular Science and Technology, funded through a NSF CRIF award (Grant No. CHE0946869) and by the Georgia Institute of Technology.

\section{SUPPORTING INFORMATION AVAILABLE}


Complete DR, UP and XP spectra together with an atomic force microscopy image of the $\mathrm{ZnO}$ surface and computational values for the hydrated defective surfaces are presented in the Supplementary Information. This information is available free of charge via the Internet at http://pubs.acs.org.

\section{REFERENCES}

(1) Koch, N. Organic Electronic Devices and their Functional Interfaces. ChemPhysChem 2007, 8, 1438-1455.

(2) Flores, F.; Ortega, J.; Vázquez, H. Modelling Energy Level Alignment at Organic Interfaces and Density Functional Theory. Phvs Chem Chem Phvs 2009, 11, 8658.

(3) Hwang, J.; Wan, A.; Kahn, A. Energetics of Metal-Organic Interfaces: New Experiments and Assessment of the Field. Mater Sci Eng R Rep 2009, 64, 1-31.

(4) Zhu, X.-Y. Electronic Structure and Electron Dynamics at Molecule-Metal Interfaces: Implications for Molecule-Based Electronics. Surf SciRep 2004, 56, 1-83.

(5) Monti, O. L. A.; Steele, M. P. Influence of Electrostatic Fields on Molecular Electronic Structure: Insights for Interfacial Charge Transfer. Phvs Chem Chem Phvs 2010, 12, $12390-12400$.

(6) Monti, O. L. A. Understanding Interfacial Electronic Structure and Charge Transfer: An Electrostatic Perspective. LPhvs Chem Lett 2012, 3, 2342-2351.

(7) Meyer, J.; Hamwi, S.; Kröger, M.; Kowalsky, W.; Riedl, T.; Kahn, A. Transition Metal Oxides for Organic Electronics: Energetics, Device Physics and Applications. Adv Mater 2012, 24, 5408-5427.

(8) Greiner, M. T.; Helander, M. G.; Tang, W.-M.; Wang, Z.-B.; Qiu, J.; Lu, Z.-H. Universal Energy-Level Alignment of Molecules on Metal Oxides. Nat. Mater 2012, 11, 76-81.

(9) Law, M.; Greene, L. E.; Johnson, J. C.; Saykally, R.; Yang, P. Nanowire Dye-Sensitized Solar Cells. Nat.Mater 2005, 4, 455-459.

(10) Li, H.; Winget, P.; Brédas, J.-L. Transparent Conducting Oxides of Relevance to Organic Electronics: Electronic Structures of Their Interfaces with Organic Layers. Chem Mater 2014, 26, 631-646.

(11) Gonzalez-Valls, I.; Lira-Cantu, M. Vertically-Aligned Nanostructures of ZnO for Excitonic Solar Cells: A Review. Enerov Envir Sci 2009, 2, 19-34.

(12) Hartel, M.; Chen, S.; Swerdlow, B.; Hsu, H.-Y.; Manders, J.; Schanze, K.; So, F. DefectInduced Loss Mechanisms in Polymer-Inorganic Planar Heterojunction Solar Cells. $\underline{A C S}$ Anol Mater Interfac 2013, 5, 7215-7218.

(13) Mattioli, G.; Dkhil, S. B.; Saba, M. I.; Malloci, G.; Melis, C.; Alippi, P.; Filippone, F.; Giannozzi, P.; Thakur, A. K.; Gaceur, M. et al. Interfacial Engineering of P3HT/ZnO Hybrid Solar Cells Using Phthalocyanines: A Joint Theoretical and Experimental Investigation. Adv Energ Mater 2014, n/a-n/a.

(14) White, M. S.; Olson, D. C.; Shaheen, S. E.; Kopidakis, N.; Ginley, D. S. Inverted BulkHeterojunction Organic Photovoltaic Device Using a Solution-Derived ZnO Underlayer. Appl Phys Lett 2006, 89. 
Grünewald et al.

(15) Komolov, A. S.; Møller, P. J.; Mortensen, J.; Komolov, S. A.; Lazneva, E. F. Electronic Properties of a Zinc Oxide Surface Modified by Ultra-Thin Layers of Conjugated Organic Molecules. Surf Sci 2005, 586, 129-136.

(16) Rangan, S.; Coh, S.; Bartynski, R. A.; Chitre, K. P.; Galoppini, E.; Jaye, C.; Fischer, D. Energy Alignment, Molecular Packing, and Electronic Pathways: Zinc(II) Tetraphenylporphyrin Derivatives Adsorbed on TiO2(110) and $\mathrm{ZnO}(11-20)$ Surfaces. $I$ Phvs Chem C 2012, 116, 23921-23930.

(17) Schlesinger, R.; Xu, Y.; Hofmann, O. T.; Winkler, S.; Frisch, J.; Niederhausen, J.; Vollmer, A.; Blumstengel, S.; Henneberger, F.; Rinke, P.; et al. Controlling the Work Function of $\mathrm{ZnO}$ and the Energy-Level Alignment at the Interface to Organic Semiconductors with a Molecular Electron Acceptor. Phvs Rev B 2013, 87, 155311.

(18) Xu, Y.; Hofmann, O. T.; Schlesinger, R.; Winkler, S.; Frisch, J.; Niederhausen, J.; Vollmer, A.; Blumstengel, S.; Henneberger, F.; Koch, N. et al. Space-Charge Transfer in Hybrid Inorganic-Organic Systems. Phvs Rev Lett 2013, 111, 226802.

(19) Winget, P.; Schirra, L. K.; Cornil, D.; Li, H.; Coropceanu, V.; Ndione, P. F.; Sigdel, A. K.; Ginley, D. S.; Berry, J. J.; Shim, J. et al. Defect-Driven Interfacial Electronic Structures at an Organic/Metal-Oxide Semiconductor Heterojunction. Adv Mater 2014, 26, 4711-4716.

(20) Vaynzof, Y.; Bakulin, A. A.; Gélinas, S.; Friend, R. H. Direct Observation of Photoinduced Bound Charge-Pair States at an Organic-Inorganic Semiconductor Interface. Phvs Rev Lett 2012, 108, 246605.

(21) Strothkämper, C.; Bartelt, A.; Sippel, P.; Hannappel, T.; Schütz, R.; Eichberger, R. Delayed Electron Transfer through Interface States in Hybrid ZnO/Organic-Dye Nanostructures. $J$ Phvs Chem C 2013, 117, 17901-17908.

(22) Němec, H.; Rochford, J.; Taratula, O.; Galoppini, E.; Kužel, P.; Polivka, T.; Yartsev, A.; Sundstrom, V. Influence of the Electron-Cation Interaction on Electron Mobility in DyeSensitized $\mathrm{ZnO}$ and TiO2 Nanocrystals: A Study Using Ultrafast Terahertz Spectroscopy. Phvs Rev Lett 2010, 104, 197401.

(23) Schulz, P.; Kelly, L. L.; Winget, P.; Li, H.; Kim, H.; Ndione, P. F.; Sigdel, A. K.; Berry, J. J.; Graham, S.; Brédas, J.-L. et al. Tailoring Electron-Transfer Barriers for Zinc Oxide/ $\mathrm{C}_{60}$ Fullerene Interfaces. Adv Func Mater 2014, 24, 7381-7389.

(24) Ozawa, K.; Mase, K. Metallization of $\mathrm{ZnO}(10-10)$ by Adsorption of Hydrogen, Methanol, and Water: Angle-Resolved Photoelectron Spectroscopy. Phvs Rev B 2010, 81, 205322.

(25) Forker, R.; Gruenewald, M.; Fritz, T. Optical Differential Reflectance Spectroscopy on Thin Molecular Films. Annu Rep Prog Chem Sect C Phvs Chem 2012, 108, 34-68.

(26) Forker, R.; Fritz, T. Optical Differential Reflectance Spectroscopy of Ultrathin Epitaxial Organic Films. Phvs Chem Chem Phvs 2009, 11, 2142-2155.

(27) Proehl, H.; Nitsche, R.; Dienel, T.; Leo, K.; Fritz, T. In situ Differential Reflectance Spectroscopy of Thin Crystalline Films of PTCDA on Different Substrates. Phvs Rev B 2005, 71, 165207.

(28) Nitsche, R.; Fritz, T. Determination of Model-Free Kramers-Kronig Consistent Optical Constants of Thin Absorbing Films from Just One Spectral Measurement: Application to Organic Semiconductors. Phvs Rev B 2004, 70, 195432.

(29) Zahn, D. R. T.; Gavrila, G. N.; Gorgoi, M. The Transport Gap of Organic Semiconductors Studied Using the Combination of Direct and Inverse Photoemission. Chem Phys 2006, $325,99-112$. 
(30) Kresse, G.; Furthmüller, J. Efficient Iterative Schemes for Ab Initio Total-Energy Calculations Using a Plane-Wave Basis Set. Phvs Rev B 1996, 54, 11169-11186.

(31) Li, H.; Schirra, L. K.; Shim, J.; Cheun, H.; Kippelen, B.; Monti, O. L. A.; Bredas, J.-L. Zinc Oxide as a Model Transparent Conducting Oxide: A Theoretical and Experimental Study of the Impact of Hydroxylation, Vacancies, Interstitials, and Extrinsic Doping on the Electronic Properties of the Polar ZnO (0002) Surface. Chem Mater 2012, 24, 3044-3055.

(32) Dienel, T.; Krause, A.; Alle, R.; Forker, R.; Meerholz, K.; Fritz, T. Alkali Metal Doped Organic Molecules on Insulators: Charge Impact on the Optical Properties. Adv Mater 2010, 22, 4064-4070.

(33) Hoffmann, M.; Schmidt, K.; Fritz, T.; Hasche, T.; Agranovich, V. M.; Leo, K. The Lowest Energy Frenkel and Charge-Transfer Excitons in Quasi-One-Dimensional Structures: Application to MePTCDI and PTCDA Crystals. Chem Phvs 2000, 258, 73-96.

(34) Forker, R.; Golnik, C.; Pizzi, G.; Dienel, T.; Fritz, T. Optical Absorption Spectra of Ultrathin PTCDA films on Gold Single Crystals: Charge Transfer Beyond the First Monolayer. Org Elec 2009, 10, 1448-1453.

(35) Gruenewald, M.; Wachter, K.; Meissner, M.; Kozlik, M.; Forker, R.; Fritz, T. Optical and Electronic Interaction at Metal-Organic and Organic-Organic Interfaces of Ultra-Thin Layers of PTCDA and SnPc on Noble Metal Surfaces. Org Elec 2013, 14, 2177-2183.

(36) Proehl, H.; Dienel, T.; Nitsche, R.; Fritz, T. Formation of Solid-State Excitons in Ultrathin Crystalline Films of PTCDA: From Single Molecules to Molecular Stacks. Phvs Rev Lett 2004, 93, 097403.

(37) Djurišić, A. B.; Fritz, T.; Leo, K. Modeling the Optical Constants of Organic Thin Films: Application to 3,4,9,10-Perylenetetracarboxylic Dianhydride (PTCDA). Opt Commun 2000, $183,123-132$.

(38) Graber, T.; Forster, F.; Schöll, A.; Reinert, F. Experimental Determination of the Attenuation Length of Electrons in Organic Molecular Solids: The Example of PTCDA. Surf Sci 2011, 605, 878-882.

(39) Hoffmann, K.; Hahn, D. Electron Spin Resonance of Lattice Defects in Zinc Oxide. Phys Stat Sol A 1974, 24, 637-648.

(40) Janotti, A.; Van de Walle, C. G. Native Point Defects in ZnO. Phvs.Rev. B 2007, 76, 165202.

(41) Heimel, G.; Romaner, L.; Brédas, J.-L.; Zojer, E. Interface Energetics and Level Alignment at Covalent Metal-Molecule Junctions: pi -Conjugated Thiols on Gold. Phvs Rev Lett 2006, 96, 196806.

(42) Oehzelt, M.; Koch, N.; Heimel, G. Organic Semiconductor Density of States Controls the Energy Level Alignment at Electrode Interfaces. Nat. Comm 2014, 5, 4174.

(43) Vázquez, H.; Flores, F.; Oszwaldowski, R.; Ortega, J.; Pérez, R.; Kahn, A. Barrier Formation at Metal-Organic Interfaces: Dipole Formation and the Charge Neutrality Level. Anol Surf Sci 2004, 234, 107-112.

(44) Temirov, R.; Soubatch, S.; Luican, A.; Tautz, F. S. Free-Electron-Like Dispersion in an Organic Monolayer Film on a Metal Substrate. Nature 2006, 444, 350-353.

(45) Forker, R.; Kasemann, D.; Dienel, T.; Wagner, C.; Franke, R.; Müllen, K.; Fritz, T. Electronic Decoupling of Aromatic Molecules from a Metal by an Atomically Thin Organic Spacer. Adv Mater 2008, 20, 4450-4454. 
Grünewald et al.

(46) Romaner, L.; Nabok, D.; Puschnig, P.; Zojer, E.; Ambrosch-Draxl, C. Theoretical Study of PTCDA Adsorbed on the Coinage Metal Surfaces, $\operatorname{Ag}(111), \operatorname{Au}(111)$ and $\mathrm{Cu}(111)$. New J Phvs 2009, 11, 053010.

(47) Hofmann, O. T.; Atalla, V.; Moll, N.; Rinke, P.; Scheffler, M. Interface Dipoles of Organic Molecules on $\operatorname{Ag}(111)$ in Hybrid Density-Functional Theory. New J Phvs 2013, 15, 123028.

(48) Zou, Y.; Kilian, L.; Schöll, A.; Schmidt, T.; Fink, R.; Umbach, E. Chemical Bonding of PTCDA on Ag Surfaces and the Formation of Interface States. Surf Sci 2006, 600, 1240 1251.

(49) Romaner, L.; Heimel, G.; Brédas, J.-L.; Gerlach, A.; Schreiber, F.; Johnson, R. L.; Zegenhagen, J.; Duhm, S.; Koch, N.; Zojer, E. Impact of Bidirectional Charge Transfer and Molecular Distortions on the Electronic Structure of a Metal-Organic Interface. Phvs Rev Lett 2007, 99, 256801.

(50) Rangger, G. M.; Hofmann, O. T.; Romaner, L.; Heimel, G.; Bröker, B.; Blum, R.-P.; Johnson, R. L.; Koch, N.; Zojer, E. F4 TCNQ on $\mathrm{Cu}, \mathrm{Ag}$, and $\mathrm{Au}$ as Prototypical Example for a Strong Organic Acceptor on Coinage Metals. Phvs Rev B 2009, 79, 165306. 
Grünewald et al.

J. Phys. Chem. C

TOC GRAPHIC

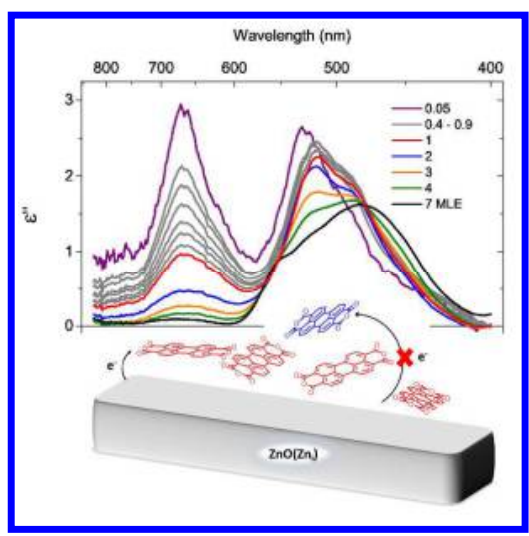

20

21

22

23

24

25

26

27

28

29

30

31

32

33

34

35

36

37

38

39

40

41

42

43

44

45

46

47

48

49

50

51

52

53

54

55

56

57

58

59

60 


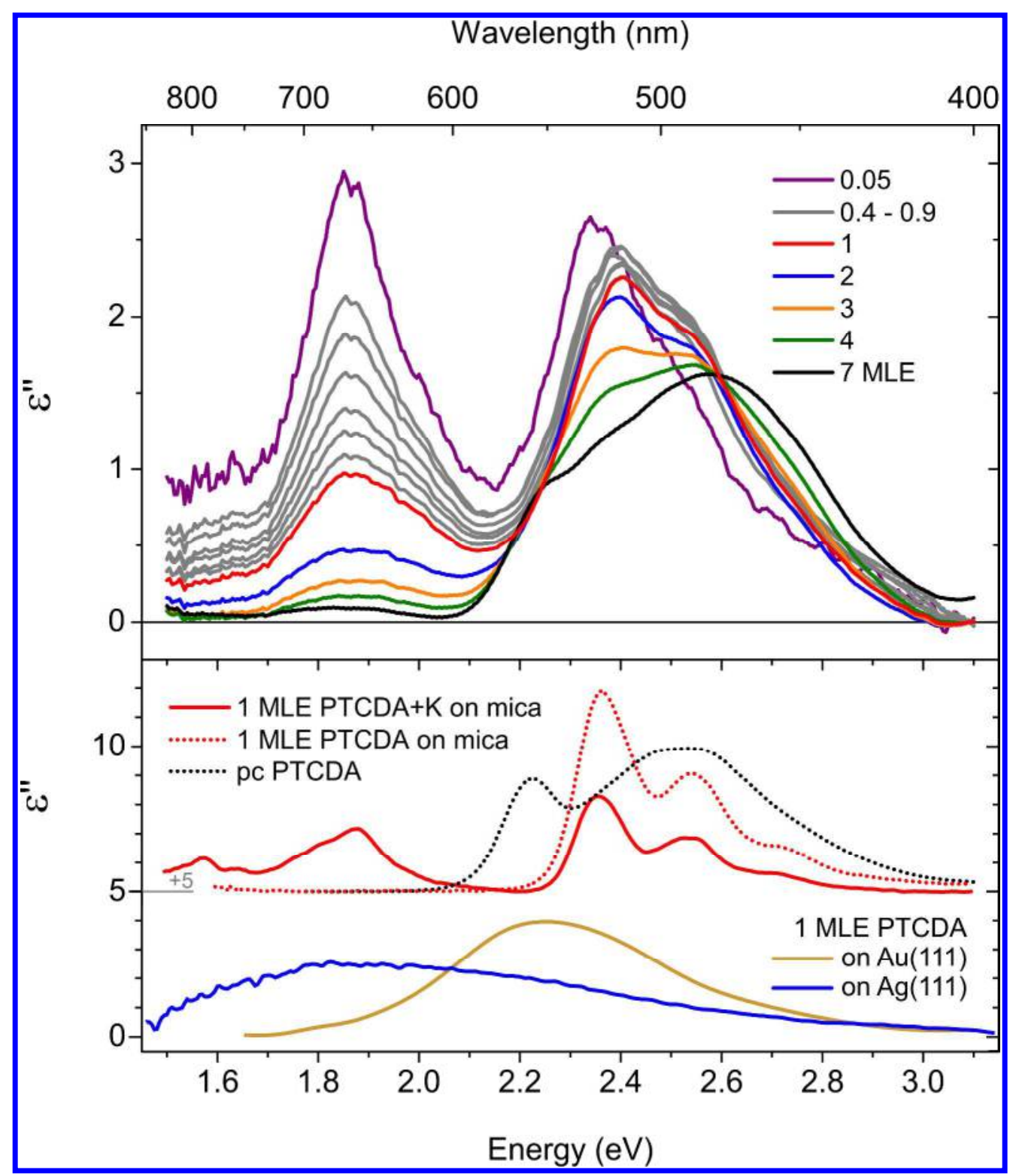

$286 \times 335 \mathrm{~mm}(300 \times 300$ DPI $)$ 


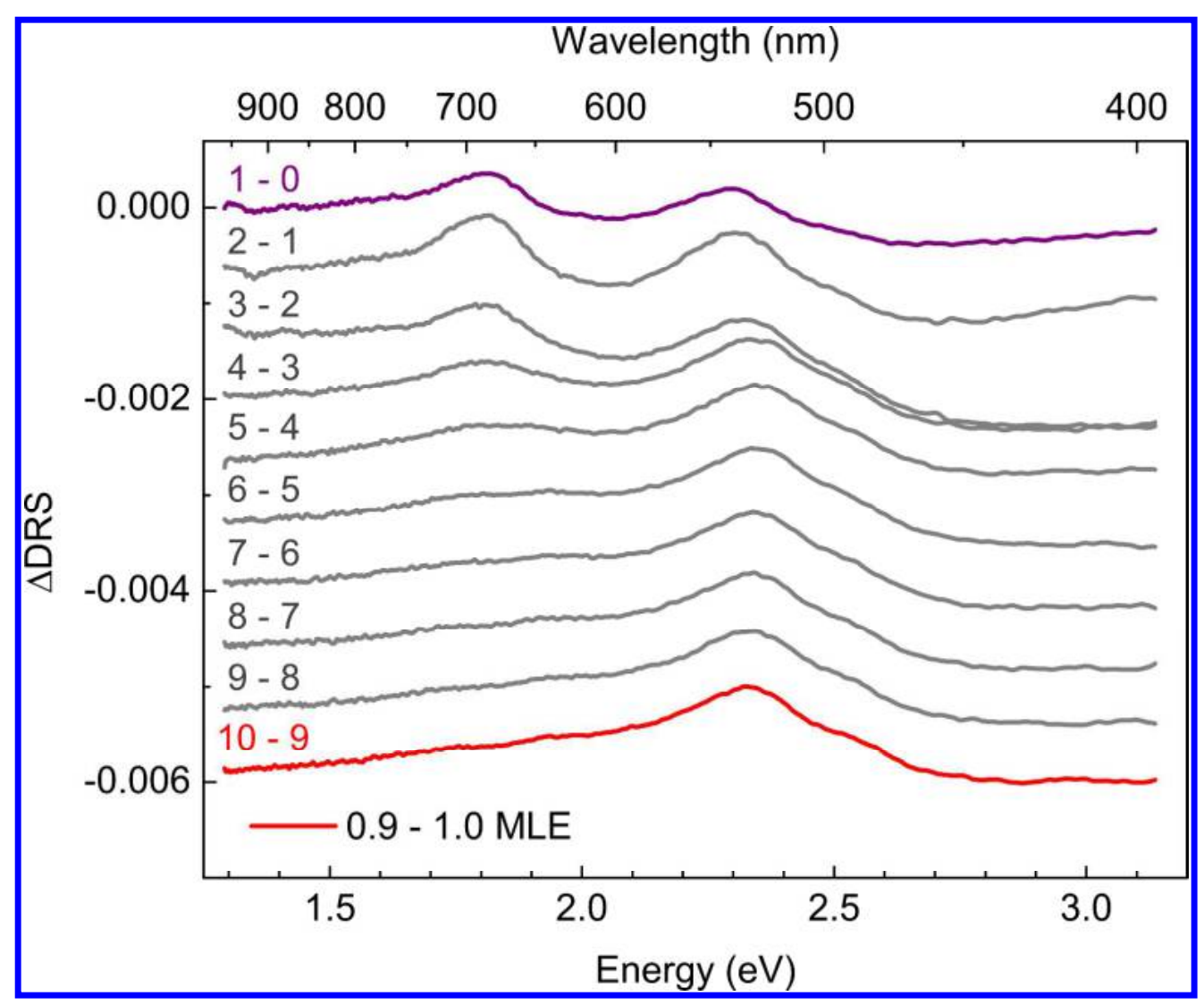

Fig. $1 \mathrm{~b}$

$282 \times 234 \mathrm{~mm}(300 \times 300$ DPI $)$ 


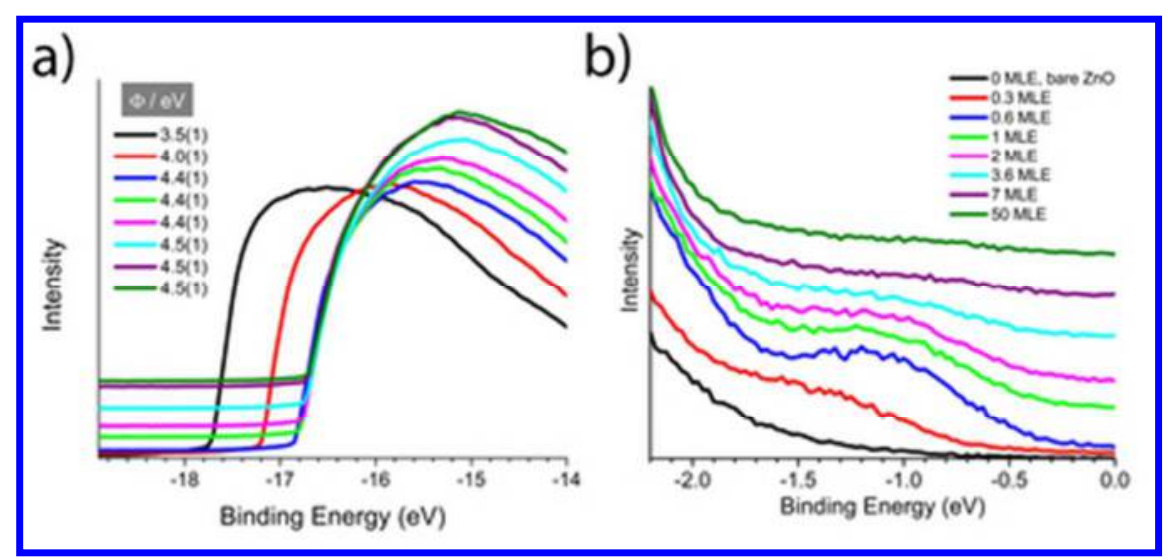

Fig. 2

$36 \times 16 \mathrm{~mm}(300 \times 300$ DPI $)$

1

2

3

4

5

6
7

8

9

10

11

12

13

14

16

17

18

19

20

2

23

25

26

27

28

29

30

31

32

33

34

35

36

37

38

39

40

41

42

43

44

45

46

47

48

49

50

51

52

53

54

55

56

57

58

59

60 


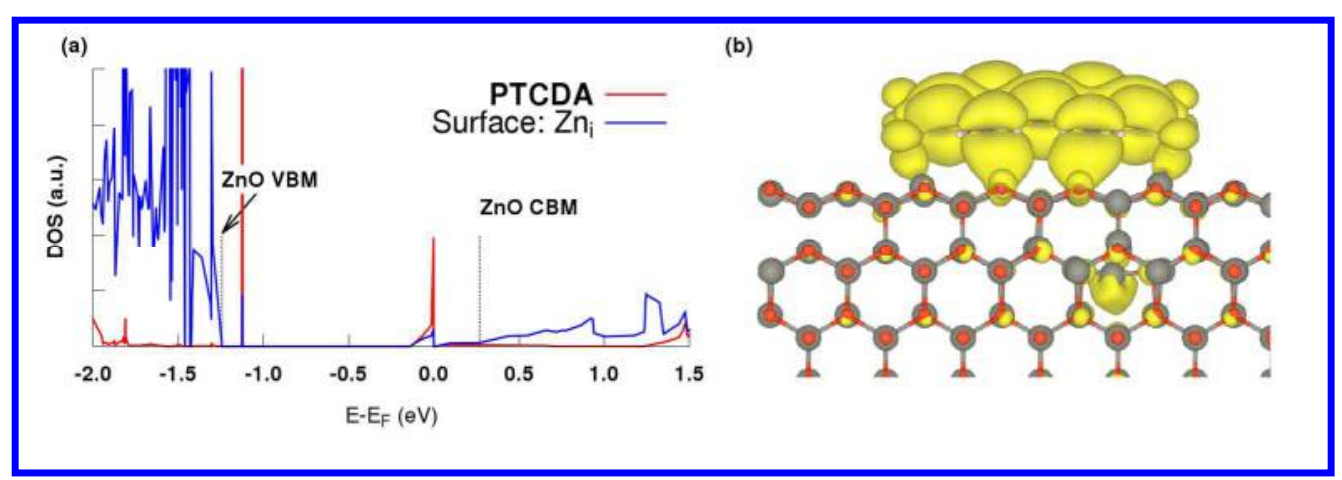

$687 \times 232 \mathrm{~mm}(72 \times 72 \mathrm{DPI})$

1

2

3

4

5

6

9

10

11

12

13

14

15

17

18

19

20

21

22

23

24

25

26

27

28

29

30

31

32

33

34

35

36

37

38

39

40

41

42

43

44

45

46

47

48

49

50

51

52

53

54

55

56

57

58

59

60 
(a)

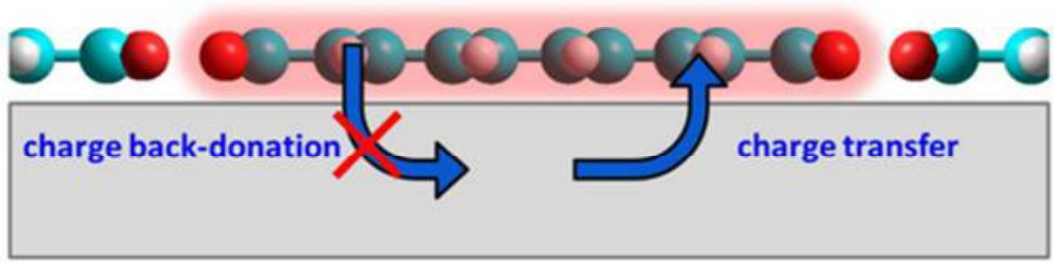

(b)

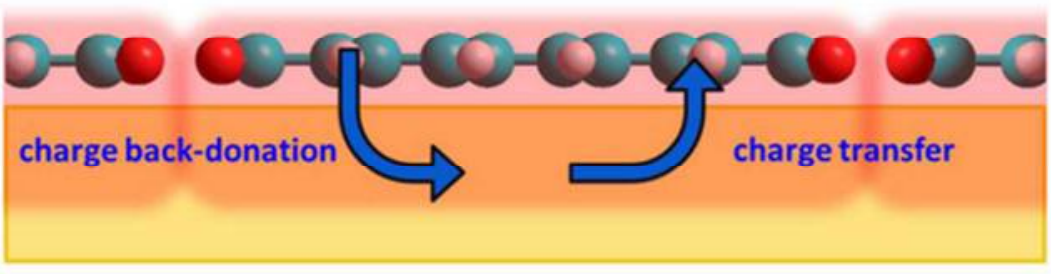

$52 \times 30 \mathrm{~mm}(300 \times 300 \mathrm{DPI})$ 


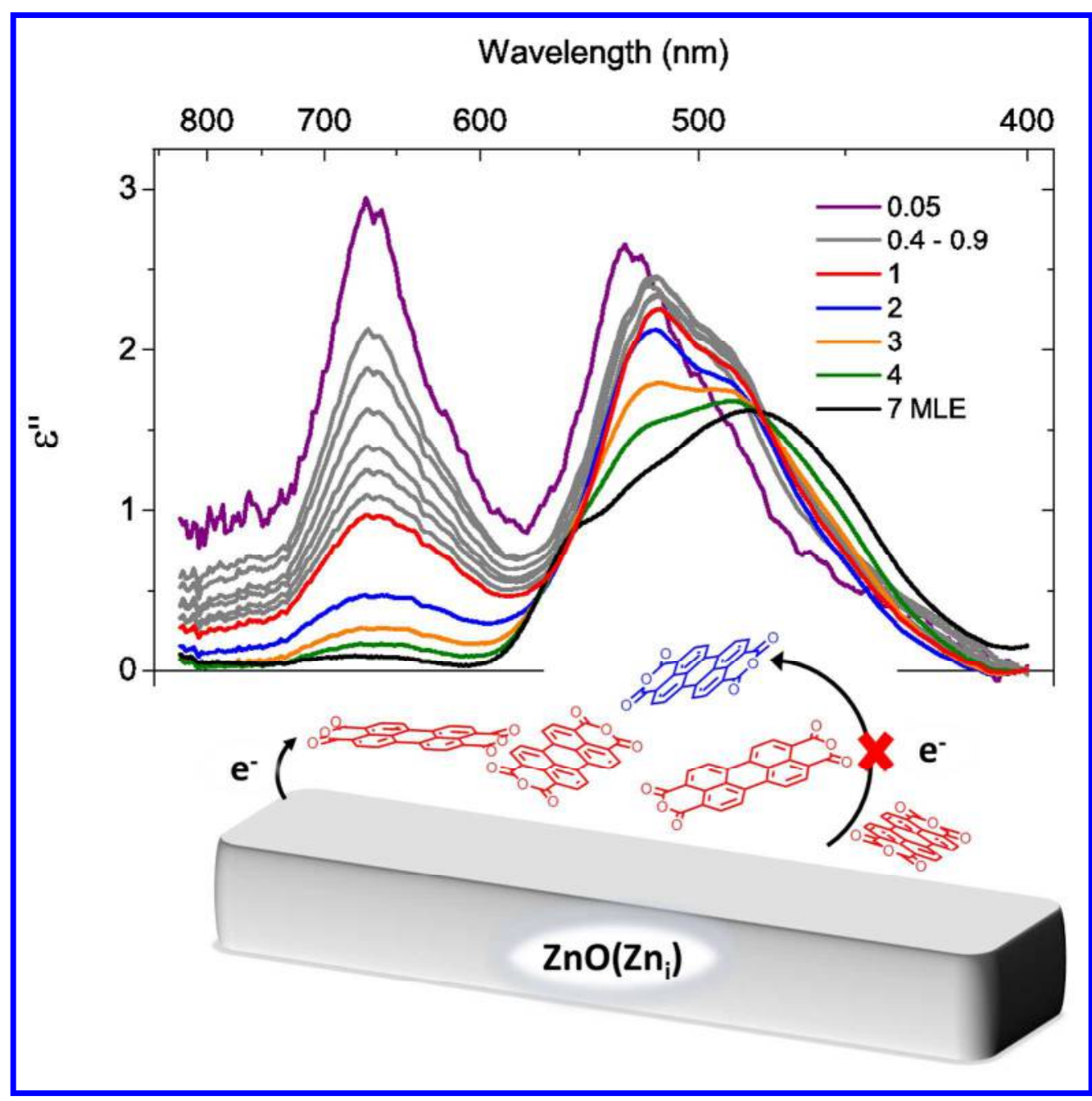

$101 \times 101 \mathrm{~mm}(600 \times 600 \mathrm{DPI})$ 